\title{
Philosophiques
}

\section{Précis de Modern Social Imaginaries}

\section{Charles Taylor}

Volume 33, numéro 2, automne 2006

URI : https://id.erudit.org/iderudit/013894ar

DOI : https://doi.org/10.7202/013894ar

Aller au sommaire du numéro

Éditeur(s)

Société de philosophie du Québec

ISSN

0316-2923 (imprimé)

1492-1391 (numérique)

Découvrir la revue

Citer ce document

Taylor, C. (2006). Précis de Modern Social Imaginaries. Philosophiques, 33(2),

477-483. https://doi.org/10.7202/013894ar d'utilisation que vous pouvez consulter en ligne.

https://apropos.erudit.org/fr/usagers/politique-dutilisation/ 


\title{
Disputatio
}

\section{Précis de Modern Social Imaginaries}

\author{
CHARLES TAYLOR \\ Northwestern University \\ Cmt111111@aol.com
}

I.

Mon livre part de la thèse qu'une des conditions préalables de la politique moderne sous toutes ses formes, soit "idéologiques ", soit "normales ", est l'invention et la diffusion d'une nouvelle conception de l'ordre normatif de la société parmi certaines élites au $\mathrm{XVI}^{\mathrm{e}}$ et XVII ${ }^{\mathrm{e}}$ siècle. C'est une conception qui a été élaborée par les théoriciens du droit naturel moderne, par exemple, Grotius, Pufendorf, ou Locke.

La société est envisagée comme étant constituée d'individus qui seraient déjà des agents moraux à part entière, capables de conclure un "contrat » qui fonde la société politique. À la différence de ses principales conceptions antérieures, celle-ci n'est pas un ordre pré-existant qui conférerait leur statut d'agent moral aux individus à partir du rôle qu'ils jouent en son sein — un statut qui serait donc différent selon le rang ou la fonction qu'ils occupent. La théorie médiévale de la monarchie française, par exemple, ou bien la conception d'une tribu constituée par une loi existant depuis l'aube des temps, nous présentent la société comme trouvant son explication en elle-même. L'agent individuel occupe la place qui lui revient, soit roi, soit noble, soit roturier, prêtre ou laïc, homme ou femme, et ne saurait s'en départir sans enfreindre l'ordre moral comme tel.

Selon la conception moderne, en revanche, l'ordre politique ne se confond pas avec l'ordre moral tout court. Il est la création des individus qui s'y soumettent. Il doit être constitué en conformité avec l'ordre fondamental, mais il ne lui est pas consubstantiel. Ce qui est fondamental, ce sont les individus, porteurs de droits, qui ont comme tels la pleine capacité d'agir et de constituer un ordre légitime.

Voilà ce qui constitue l' " atomisme » de l'ordre moral moderne. Cette primauté de l'individu a deux facettes. La société politique est créée par les hommes, et elle a comme finalité de les servir. C'est-à-dire, de défendre leurs droits et de servir les buts qu'ils se proposent, chacun pour soi. Ces buts englobent essentiellement la conservation et la recherche des moyens à cette fin. En dehors de la défense contre l'aggression, ces moyens comprennent principalement la production des biens nécessaires à la vie. 
II.

Il s'agit donc d'une conception de l'ordre qui est : a) atomiste, b) centrée sur les droits, en particulier de liberté, c) et qui accorde une importance sans précédent à l'action économique. En un sens, je prends l'invention de cette idée d'ordre et sa diffusion parmi les élites de l'époque comme point de départ de ma discussion. Ce qui soulève toutes sortes de questions, bien entendu.

Pourtant, je crois que l'on peut placer l'avènement de cette idée dans le contexte d'un certain nombre de lignes de force de la modernité naissante. Ce qui, à défaut de fournir une explication, nous la rendrait toutefois moins suspecte comme hypothèse.

L'atomisme est un aspect connu de la modernité, mais on peut se demander pourquoi il a surgi précisément à ce moment-là de notre histoire. On peut l'envisager comme le point culminant d'un long processus, celui du "désenchâssement " de l'individu. Il est clair que la conscience humaine la plus primitive, comme on la voit dans les religions les plus anciennes, comportait un triple enchâssement. L'individu ne se concevait pas sans la société, qui, elle, se définissait en relation avec le cosmos, lequel était à son tour saturé de sacré. C'est la période " axiale » qui aurait ébranlé cette chaîne d'emboîtements ${ }^{1}$. Il ouvrait une nouvelle possibilité, celle d'une pratique métaphysique ou religieuse individuelle, distincte des grands actes du culte public.

Les grandes civilisations qui naissaient des révolutions axiales subissaient toutes la tension entre deux formes de vie religieuse, les cultes de la cité, du village ou de la famille, d'une part, qui visaient souvent le maintien de la vie et du bien-être; et, d'autre part, les modes de contemplation, de dévotion, ou de discipline que des individus, souvent associés dans des collectivités nouvelles, pouvaient pratiquer dans le but de transcender la vie de tous les jours. Malgré les tentatives de rendre ces deux formes complémentaires — par exemple: «le clergé prie pour tous, les nobles combattent pour tous, les paysans labourent la terre pour tous »-, les tensions persistaient.

Pourtant, la société entière continuait à se concevoir sur le modèle de l'enchâssement. Le moine, le bhikhu, le philosophe, poursuivait une vocation individuelle, mais il restait un « individu hors du monde », pour emprunter l'expression de Louis Dumont ${ }^{2}$. C'est dire que le "monde ", l'espace que nous partageons, était encore conçu sur le modèle de l'ordre qui est antérieur aux individus, d'où ils tirent leur identité, suivant leur rang, leur rôle ou leur fonction.

Le grand désenchâssement arrive au moment où le monde lui-même est reconceptualisé à partir de la religion de l'élite, et non plus dans les formes qui remontent aux origines. Il arrive quand le compromis, ou l'équilibre instable des civilisations post-axiales, est rompu en faveur de la religion

1. Je parle de l'» Achsenzeit » de Karl Jaspers. Voir Vom Ursprung und Ziel der Geschichte, Munich, Piper 1988.

2. Voir Louis Dumont, Essais sur l'individualisme, Paris, Seuil, 1983, pp. 33-67. 
désormais définie comme la seule vérité. Cette rupture fut réalisée dans la chrétienté latine vers la fin du moyen âge, début de l'ère moderne, par les réformes, et de la façon la plus évidente par LA Réforme, avec le but avoué d'en finir avec un christianisme à plusieurs vitesses. Désormais, il n'y avait plus de vocations spéciales, de perfection. Tout le monde devait être chrétien à part entière, ou bien exclu, sur le chemin de la damnation.

En clair, mon hypothèse, c'est que, pendant la première modernité, les tentatives de réaliser un christianisme à une seule vitesse, les nouvelles disciplines, les nouveaux modes d'organisation sociale, ecclésiale, politique, constitués à cette fin et animés en partie aussi par le néo-stoïcisme, le désenchantement de la nature et la réorganisation de la société, tout cela a fait basculer la conscience des élites hors des cadres séculaires de l'enchâssement social-cosmique-sacré. Il en résulta une nouvelle idée de l'ordre moral, qui était d'autant plus bienvenue qu'elle semblait offrir un principe d'ordre en dehors de la partisanerie confessionnelle. En un certain sens, ce nouvel ordre moral appartient à la fois à l'histoire de la démocratie et à celle de la sécularité.

Un autre aspect de cet ordre est l'importance accordée à l'action économique. Cela s'inscrit dans la nouvelle affirmation de la "vie ordinaire ", de la production et de la reproduction, qui marque la modernité naissante ${ }^{3}$.

III.

Voilà pour la conception de l'ordre moral moderne. Mais elle ne nous intéresse ici que dans la mesure où elle permet de comprendre les développements politiques de l'ère révolutionnaire. Bien sûr, il est clair que cette conception a influencé la théorie politique; on pourrait dire qu'elle est déjà une théorie politique, dans les écrits de Grotius ou de Locke, par exemple. Et on a vu comment, plus tard, elle a pu se marier avec des théories plus anciennes et les modifier profondément ; c'est le cas de la version de Rousseau qui s'est exprimée comme la forme renouvelée de l'humanisme civique, de la récupération de la république et de la vertu antiques.

Toutefois, pour avoir son plein effet sur la politique de notre ère, la conception de l'ordre a dû déborder le domaine de la théorie pour entrer dans l'imaginaire social. Comment expliquer cette distinction ${ }^{4}$ ? Je note trois contrastes :

— Les théories sont souvent le fait d'individus isolés, elles peuvent circuler dans des milieux restreints de penseurs; l'imaginaire social est partagé par la société entière, ou, du moins, par des couches importantes de la société. chap. 13.

3. J'ai traité de ce développement dans Les Sources du Moi, Paris, Le Seuil, 1999,

4. J'emprunte le terme « imaginaire social » à Cornelius Castoriadis, quoique je l'utilise en un sens légèrement différent du sien. 
- Une théorie s'exprime dans le médium de la pensée, la prose déclarative, en des énoncés qui sont censés obéir aux exigences de la logique et des formes d'arguments reconnus en philosophie; l'imaginaire, en revanche, s'exprime aussi à travers la gamme entière des registres, dans des contes, des légendes, des vies reconnues comme paradigmatiques, des modes d'adresse, des pratiques sociales, voire des pratiques quotidiennes, du genre de l'habitus dont parle Bourdieu.

- Cela est vrai parce que l'imaginaire social constitue en fait l'ensemble de la compréhension partagée, souvent implicite, d'une population, qui est sous-jacente à leurs pratiques communes. C'est ce qu'ils doivent «imaginer» ensemble pour que leurs pratiques aient le sens qu'elles ont. Par exemple, quand, le jour de l'élection, nous allons chacun dans l'isoloir pour cocher une case sur un bulletin, cela n'a de sens que parce que nous concevons une décision collective, engageant des millions de concitoyens, comme émergeant d'une multitude de décisions ponctuelles. Cela ne va pas de soi; pour la plupart des sociétés pré-modernes une décision collective engendrée de la sorte paraîtrait assez bizarre sinon contre nature; il faudrait plutôt un consensus qui serait sorti d'échanges intenses conduits face à face. C'est pourquoi la démocratie moderne, dans des sociétés de taille énorme, quasi-impériale, a passé longtemps pour impossible. Il fallait que se développe tout un ensemble de conceptions - certaines tacites - de l'individu, du citoyen, des rapports possibles avec des milliers de gens que l'on ne rencontrera jamais mais qui font partie du même "peuple", pour que cette forme de légitimité naisse, que nous tenons pour acquis, pour le sens commun le plus évident.

Dans l'expression « imaginaire social », le terme «social» figure doublement, comme adjectif subjectif et objectif. C'est notre façon partagée d'imaginer la société.

On peut lier le concept d'imaginaire social à celui de répertoire. Partager une certaine compréhension commune rend possible, pour une population donnée, de s'engager dans certaines pratiques, comme c'est le cas pour la démocratie à grande échelle invoquée plus haut, avec ses élections et ses systèmes de représentation. Avec l'évolution de l'imaginaire, le répertoire s'enrichit (et dans d'autres dimensions peut bien s'appauvrir aussi, bien entendu). La nature et l'étendue du répertoire est une question cruciale pour les transformations révolutionnaires; nous y reviendrons bientôt.

\section{IV.}

Or, l'histoire moderne, c'est en un sens la pénétration de notre imaginaire social par la conception de l'ordre que nous avons décrite plus tôt. Cette conception a pour ainsi dire colonisé et transformé les imaginaires précédents, qui étaient tous plus holistes, et soit hiérarchiques, soit égalitaires, mais animant des sociétés de petite taille et locales. 
Je distingue trois figures majeures de l'imaginaire social moderne qui porte l'empreinte de l'ordre moral. D'abord, la conception d'une "économie ».

Un des premiers fruits de la nouvelle conception de l'ordre était les théories de l'économie moderne, dont les physiocrates ont proposé une première version, suivis de près par Adam Smith. Elles conçoivent la dimension économique de la société comme régie par ses propres lois. L'économie est une série de flux circulaires, de biens, de produits, d'argent, qui ont leur propre logique interne et suivent des lois propres à son domaine. L'élément «nomos » dans l'oikonomia traditionnelle référait à l'ordre que l'oikonomikos, le gestionnaire, imposait à son domaine. Dans la nouvelle conception, le nomos est une loi de la nature, comme celles que Newton a découvertes dans l'univers des corps matériels.

Cette façon de voir transforme le domaine social, ou plutôt constitue ce domaine pour la première fois. Dans la plupart des sociétés prémodernes, la société n'avait d'autre conception d'elle-même comme un tout que celle qui l'envisageait comme structure politique. Elle était constituée comme société par sa loi, ou par les fonctions essentielles identifiées par cette loi : celle du roi pour une monarchie, par exemple. La France n'était pas la France sans son roi. Dans la chrétienté, il fallait ajouter un autre principe d'ordre : l'Église. Mais, dorénavant, la société se conçoit comme tout à partir d'une pluralité de perspectives. L'économie n'est que la première à s'émanciper. Divers aspects de la vie sociale sont reconnus, étudiés, parfois défendus, comme suivant leur propre logique interne, bonne ou mauvaise, bénéfique ou dangereuse. La politique est profondément relativisée, suivant la logique de l'ordre moral moderne lui-même. Car celui-ci non seulement accordait une importance sans précédent à la dimenson économique, comme nous l'avons constaté plus haut, mais il définissait le politique comme réalité non pas primaire, mais essentiellement dérivée. Le pouvoir politique était la création des individus, et cela comme instrument au service de leur projet essentiel d'auto-conservation.

C'est grâce à cette émancipation de la "société » que l'on commence à parler à cette époque de la "société civile » comme réalité indépendante de l'État. La dimension économique est la première mais non pas la seule facette de ce nouvel interlocuteur du pouvoir. Or, si tout cela se jouait d'abord sur le plan de la théorie, au XVIII e siècle l'imaginaire social, au moins celui des élites, ne tarda pas à être pénétré et à se transformer. La vie politique en fut profondément bouleversée.

Pour illustrer ce dernier point, je voudrais relever une autre figure de l'imaginaire social moderne : la sphère publique. On peut mesurer non seulement la modification de l'imaginaire social, mais aussi son énorme pouvoir créateur si on suit l'avènement de l'" opinion publique " comme force politique. Dans le courant du XVIII ${ }^{\text {e }}$ siècle, en Angleterre, en France, dans les colonies anglaises de l'Amérique, l'opinion publique se constitue lentement, d'abord comme point de référence, et enfin comme source de légitimité. Cette nouvelle force est inséparable d'une nouvelle conscience collective. 
Pour parler de l'opinion publique, il fallait reconceptualiser un ensemble d'activités - circulations d'écrits, de livres, de pamphlets, discussions entre petits groupes dans des salons, "coffee houses ", réceptions, que ces écrits inspiraient, et les nouveaux écrits qui en naissaient - comme un seul processus de délibération commune, d'où il devait sortir un résultat, un consensus. C'est comme si on ramassait toutes ces activités éparses et lointaines pour les réunir conceptuellement dans l'analogue d'une assemblée délibérative, telles les réunions dans le forum romain d'antan, assemblées capables d'engendrer une quasi-décision, une opinion majoritaire.

Ce n'est pas moi qui importe cette image du forum de l'extérieur pour décrire ce phénomène. Car la reconceptualisation se faisait en une série d'images, dont celle-ci. Elle pouvait être invoquée explicitement ( «le forum de l'opinion publique»), ou implicitement - comme quand Trenchard s'adresse au public anglais pour critiquer la corruption des mœurs politiques en signant ses articles du nom de "Caton ", le défenseur austère des mœurs républicaines qui, lui, parlait de vive voix à ses concitoyens. Une autre image, très invoquée en France (il n'est peut-être pas difficile de comprendre pourquoi) est celle du tribunal ${ }^{5}$. L'importance des images ici provient du fait qu'à la différence de l'économie, où la nouvelle façon de voir s'exprime d'abord dans une théorie pour envahir ensuite l'imaginaire social, la sphère publique semble naître d'une transformation de l'imaginaire même. C'est dans leur façon d'envisager et de traiter cet ensemble de discussions et d'échanges épars que les élites du XVIII ${ }^{\mathrm{e}}$ siècle se sont découvertes et constituées comme porteuses d'une "opinion publique » ayant finalement force de légitimité. La mutation de l'imaginaire a créé une nouvelle force politique, une instance habilitée à conférer la légitimité aux actes du gouvernement. Rien n'est plus extraordinaire que cette évolution des lignes de force de la politique française pendant les trois ou quatres décennies qui ont précédé la Révolution. Au début de cette période, le régime se donnait comme but de contrôler les opinions de ses citoyens, comme amas d'individus épars, par les mesures de censure, permis de publications, et le reste. Passant par une phase intermédiaire où le gouvernement commence à intervenir pour former l'opinion, on finit avec la capitulation du gouvernement royal à la veille de la Révolution devant l'opinion, maintenant devenue force collective irrésistible, par exemple dans le rappel de Necker. (Évidemment, le fait que cette opinion pouvait affecter la disponibilité de fonds d'emprunt pour combler le déficit n'était pas étranger à son nouveau pouvoir.)

À la différence de l'économie, la sphère publique change la donne dans le domaine politique. Elle accorde déjà son sens à l'idée de " peuple », capable de fonctionner comme agent collectif en dehors de son organisation politique. «L'opinion publique » était censée exister en dehors du pouvoir, en face de lui, lui conférant en fin de compte sa légitimité, ou la lui retirant. Mais cette

5. Citation de Malesherbes. 
idée du peuple comme agent collectif fondamental, indépendant des structures politiques du moment, et source de légitimité, est à un cheveu de l'idée révolutionnaire de base: que les peuples sont souverains, qu'ils ont donc le droit de faire et de défaire des régimes. C'est cette troisième figure de l'imaginaire moderne qui est le fruit ultime de ce déplacement, cette démotion du politique, inscrite dans l'ordre moral moderne. Les structures politiques, en tant que réalités dérivées et instrumentales, sont à la merci d'agents qui se constituent en dehors du politique, parce qu'ils constituent plutôt sa source.

Les grandes révolutions, américaine et française, marquent le moment et le chemin par lesquels nous avons franchi cette dernière étape, pour faire de la souveraineté des peuples le principe majeur de notre civilisation. C'est pourquoi elles constituent un objet privilégié de notre historiographie et vont continuer de le faire jusqu'au moment où ce principe disparaîtra ou bien deviendra si peu problématique que nous n'aurons plus à y penser; autrement dit, dans un avenir indéterminé.

Dans un chapitre important de mon livre, j'essaie de tracer les chemins et les modalités par lesquels l'ordre moral moderne est entré dans l'imaginaire social pour construire cette troisième figure, en comparant les deux grandes révolutions du XVIII ${ }^{\mathrm{e}}$ siècle.

Cette tentative de définir les formes centrales de l'imaginaire social moderne en Occident devrait nous aider à mieux saisir les différentes formes de modernité qui se constituent de nos jours en dehors de l'Occident. Les différences s'appréhendent plus clairement par le contraste des imaginaires sociaux; c'est du moins la thèse fondamentale de cette œuvre. 